\title{
Early Physics with the LHCf Experiment at LHC
}

A. Tricomi ${ }^{\ddagger}$, O. Adriani $^{b}$, L. Bonechi ${ }^{b}$, M. Bongi $^{c}$, G. Castellini $^{d}$, R. D’Alessandro ${ }^{b}$, K. Fukui ${ }^{e}$, M. Hagenauer ${ }^{f}$, Y. Itow ${ }^{e}$, K. Kasahara ${ }^{g}$, K. Kawade ${ }^{e}$, D. Macina ${ }^{h}$, T. Mase ${ }^{e}$, K. Masuda ${ }^{e}$, Y. Matsubara ${ }^{e}$, H. Menjo ${ }^{c}$, G. Mitsuka ${ }^{e}$, M. Mizuishi ${ }^{g}$, Y. Muraki ${ }^{i}$, M. $^{2}$

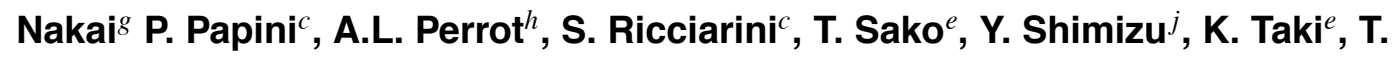

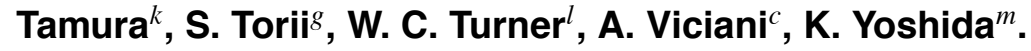

${ }^{a}$ University of Catania and INFN Catania, Italy

${ }^{b}$ University of Firenze and INFN Firenze, Italy

${ }^{c}$ INFN Firenze, Italy

${ }^{d}$ IFAC-CNR and INFN Firenze, Italy

e Solar-Terrestrial Environment Laboratory, Nagoya University, Nagoya, Japan

${ }^{f}$ École-Polytechnique, Paris, France

${ }^{g}$ Research Institute for Science and Engineering, Waseda University, Tokyo, Japan

${ }^{h}$ CERN, Geneva, Switzerland

${ }^{i}$ Konan University, Kobe, Japan

${ }^{j}$ Institute for Cosmic Ray Research, University of Tokyo, Kashiwa, Chiba, Japan

${ }^{k}$ Kanagawa University, Yokohama, Japan

${ }^{l}$ LBNL, Berkeley, California, USA

${ }^{m}$ Shibaura Institute of Technology, Saitama, Japan

E-mail: alessia.tricomi@ct.infn.it, lhcf_all@stelab.nagoya-u.ac.jp

LHCf, the smallest of the LHC experiment, has been designed to study the forward production of neutral particles in proton-proton collisions at LHC. The aim of the experiment is to provide valuable information for the calibration of the nuclear interaction models used in the Monte Carlo codes developed for high and ultra-high energy cosmic ray Physics. These data are of extreme importance for the extraction of primary cosmic ray properties. In particular, since LHCf will take data already in the first phase of operation of the LHC at 3.5+3.5 TeV up to the ultimate energy of $7+7 \mathrm{TeV}$, it will be able to span the most interesting energy range for high energy cosmic rays, above the "knee" region. To accomplish these precision measurements, LHCf is made of two double arm high precision calorimeters placed on both side of ATLAS interaction point. In this paper the performance of the LHCf detector will be discussed with particular emphasis to the early physics program.

European Physical Society Europhysics Conference on High Energy Physics, EPS-HEP 2009, July 16 - 222009

Krakow, Poland

\footnotetext{
* Speaker.
} 


\section{Introduction}

LHCf is a LHC experiment designed to study neutral particles produced in proton-proton collisions at LHC in the very forward region. The detector consists of two sampling and imaging calorimeters placed at $\pm 140 \mathrm{~m}$ from ATLAS interaction point (IP1) inside the zero-degree neutral absorbers (Target Neutral Absorber, TAN). Charged particles from the IP are swept away by the inner beam separation dipole before reaching the TAN, so that only photons mainly from $\pi^{0}$ decays, neutrons and neutral kaons reach the LHCf calorimeters. LHCf covers the pseudorapidity range from 8.4 to infinity.

The two calorimeters have a double tower structure. Each tower is made by 16 layers of plastic scintillators interleaved by tungsten layers as converter. Additionally, a set of four X-Y position sensitive layers provide incident shower positions, in order to obtain the transverse momentum of the incident primary and to correct for the effect of leakage from the edges of the calorimeters.

While the two calorimeters are identical for the calorimetric structure, they slightly differ for the geometrical arrangement of the two towers and for the position sensitive layers made by $1 \mathrm{~mm}^{2}$ scintillating fibers in one calorimeter and silicon micro-strip layers in the other. A detailed description of the LHCf detector can be found in [1].

Differently from the other LHC experiments, the main physics motivations of LHCf are mostly related to the astroparticle physics. LHCf capability to perform measurements of neutral particles spectra is a key ingredient to better understand many properties of primary cosmic rays, like the energy spectrum and the composition, whose knowledge is finally strictly related to our capability to understand the origin of high energy phenomena in the Universe. Dedicated extensive air shower experiments are in place since many years and have strongly contributed to our understanding of High and Ultra High Energy Cosmic (UHECR) Ray Physics. However, the results of these experiments are in some cases not fully in agreement and, in addition, the interpretation of their data in terms of primary cosmic ray properties is strongly affected by the knowledge of the nuclear interactions in the earth's atmosphere. This is true, for instance, for the interpretation of the behaviour of the energy spectrum in the UHE region, in particular the existence of events above the so called GZK cut-off, and the chemical composition of cosmic rays.

The possible existence of cosmic rays beyond the GZK cut-off (with energy above $10^{80} \mathrm{eV}$ ) has great relevance as their origin, propagation and interactions are unknown and may yield information about new physics. Indeed, evidence of UHECR, above the GZK cut-off, has been reported for the first time by the AGASA experiment [2]. On the contrary, the results of the HiRes [3] experiment and, more recently, the ones of the Pierre Auger Collaboration [4] are consistent with the existence of the cut-off. The disagreement among data would be reduced by adjusting the energy scales of the different experiments to account for systematic effects in the determination of the particle energy, that might be due to different detecting techniques. Similar considerations hold for the interpretation of cosmic ray composition since it is directly related to their primary sources. Auger has reported a correlation between the distribution of active galactic nuclei (AGN) and the arrival direction of the events above $6 \times 10^{19} \mathrm{eV}$ [5], which can travel through nearby inter-galactic space almost without any deviation by magnetic fields. While this would naturally suggests that the primary cosmic rays are most likely protons, other measurements reported by Auger based on shower elongation [6] indicate that the composition is likely dominated by heavy particles. Because the 
deduction of primary cosmic ray composition from the elongation parameter has a strong model dependence, especially in the highest energy region, the reduction of the uncertainty in the interaction models is important for solving this puzzle. The correct interpretation of the results of such cosmic-ray experiments depends strongly on the use of Monte Carlo codes, which are necessary to simulate the nuclear-cascade showers produced by the primary particles when interacting with the atmosphere, and to determine their energy and composition. Accelerator experiments validating the interaction model chosen are hence essential. As a matter of fact air shower development is dominated by the forward products of the interaction between the primary particle and the atmosphere. The only available data on the production cross-section of neutral pions emitted in the very forward region have been obtained more than twenty years ago by the UA7 Collaboration [7] at the CERN SppsS up to an energy of $10^{14} \mathrm{eV}$ and in a very narrow pseudo-rapidity range. The LHCf experiment at LHC has the unique opportunity to take data at energies ranging from $\sqrt{s}=7 \mathrm{TeV}$ up to $14 \mathrm{TeV}$ thus extending significantly the energy range up to a region of great interest for high energy cosmic rays, the region between the "knee" and the GZK cut-off.

\section{Physics Performances}

The LHCf detector performance has been carefully studied using both simulations and beam test data. Tests at the CERN SPS were done on the final detectors to calibrate the calorimeters and finally measure their performances in terms of energy resolution and spatial resolution of the sensitive layers, by using muon, electron and hadron beams. The measured energy resolution for the calorimeters is better than $4 \%$ at $200 \mathrm{GeV}$ (see Fig. 1), while the position resolution in locating the shower center for particles above $100 \mathrm{GeV}$ (which is the region of interest for LHCf) is about $200 \mu \mathrm{m}$ for scintillating fibre layers (ARM1) and about $50 \mu \mathrm{m}$ for the silicon micro-strip layers (ARM2). In Fig. 2 the energy dependence of the measured silicon spatial resolution both for $\mathrm{X}$ and $\mathrm{Y}$ view is shown. Electrons of energies ranging from $50 \mathrm{GeV}$ up to $200 \mathrm{GeV}$ have been considered. The measured resolution is in agreement with simulation expectations and well satisfies the design requirement [8].

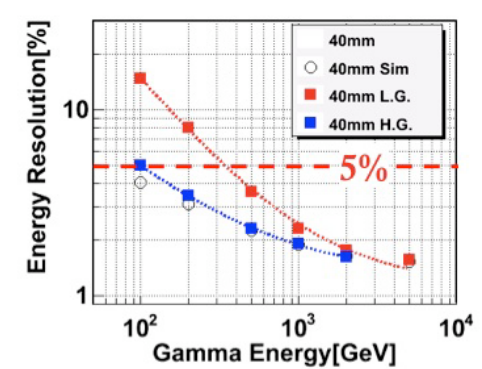

Figure 1: Energy resolution of ARM1 detector (4 $\mathrm{mm} \times 4 \mathrm{~mm}$ tower). Test beam results (red/blue full marker) are in good agreement with simulation results (white open marker). Data have been taken with two different PMT HV, corresponding to two different gain conditions.

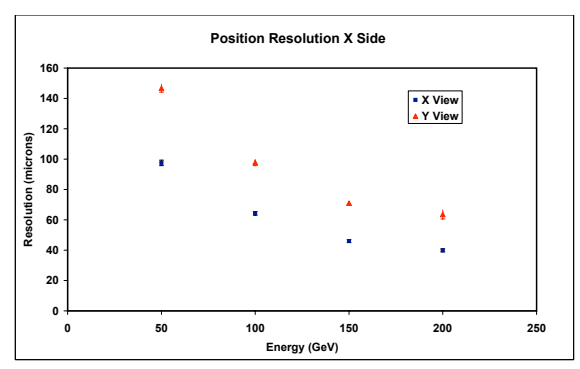

Figure 2: ARM2 silicon detector position resolution as function of energy. Resolution of $\mathrm{X}$ and $\mathrm{Y}$ view is shown. 
In order to calibrate the Monte Carlo codes a precision measurement of the energy scale is needed. For this reason LHCf relies on a very precise reconstruction of the $\pi^{0}$ mass, by reconstructing in the two towers the showers from the $2 \gamma$ from $\pi^{0}$ decays. The $\pi^{0}$ mass reconstruction capability of the experiment was also investigated during beam test, by placing a 60 -mm thick carbon target in front of ARM1 on a $350 \mathrm{GeV}$ proton beam. Results are reported in Fig. 3: the peak is clearly visible and can be used for the absolute calibration of the energy scale of the experiment.

A detailed study on $\pi^{0}$ reconstruction has been carried on also with simulation. MC simulations

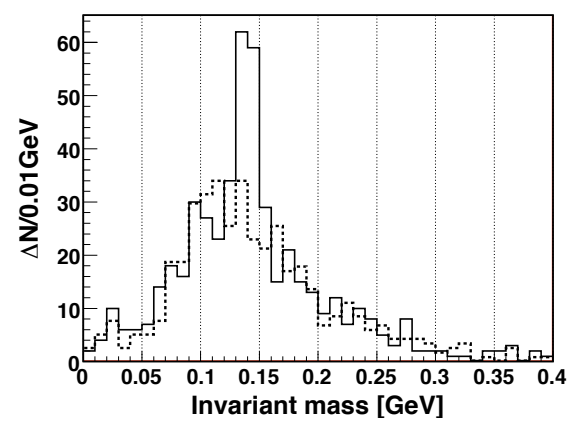

Figure 3: Reconstructed photon pair invariant mass distribution at the SPS beam test. The peak at the mass of the $\pi^{0}$ is clearly visible (the dotted histogram is the background calculated using uncorrelated photon pairs).

for $1.04 \times 10^{7}$ and $1.17 \times 10^{7} \mathrm{p}$-p collisions, each corresponding to about 20 minutes operation during the LHC beam commissioning with 43 bunches and $10^{29} \mathrm{~cm}^{-2} \mathrm{~s}^{-1}$ of luminosity, were carried out with the DPMJET3.03 model for two different detector position (one in which the detector is in the nominal position and one in which the detector is $10 \mathrm{~mm}$ down), respectively. With a set of cuts and optimization for background rejection (for analysis details see Ref. [9]), the reconstructed $\pi^{0}$ energy spectrum well reproduced the original production spectrum, as can be seen in Fig. 4. A good discrimination between different Monte Carlo models is hence feasible. The models used are DPMJET3 [10], QGSJET-II [11], SYBILL [12] and EPOS1.99 [13].

Fig. 5 shows LHCf expectations for the $\gamma$ and neutron energy spectra. Depending on the nuclear interaction model used the energy spectra change more or less significantly. In the case of $\gamma$ energy spectra, the discriminating power is already significant at $1 \mathrm{TeV}$ between SYBILL and the other codes, while discrimination between DPMJET3 and QGSJET-II can be achieved through a more sophisticated analysis, as described in Ref. [8].

The neutron sample gives more discrimination between different models. As it is shown in Fig. 5, even with a pessimistic 30\% energy resolution, a very good disentangling of the different models is feasible.

Since the LHCf experiment will collect data already at the beginning of LHC beam commissioning phase, recently we are evaluating the Physics performance for different $p$-p center of mass energy. In Fig. 6 an example for $\gamma$ and neutron energy spectra for one minute exposure at $10^{9}$ $\mathrm{cm}^{-2} \mathrm{~s}^{-1}$ with $5+5 \mathrm{TeV}$ center of mass energy is shown. As can be seen from this plot (which does not take into account detector resolution), the LHCf experiment will be able to disentangle different interaction models already at lower energy and with very low statistics, thus ensuring a calibration 

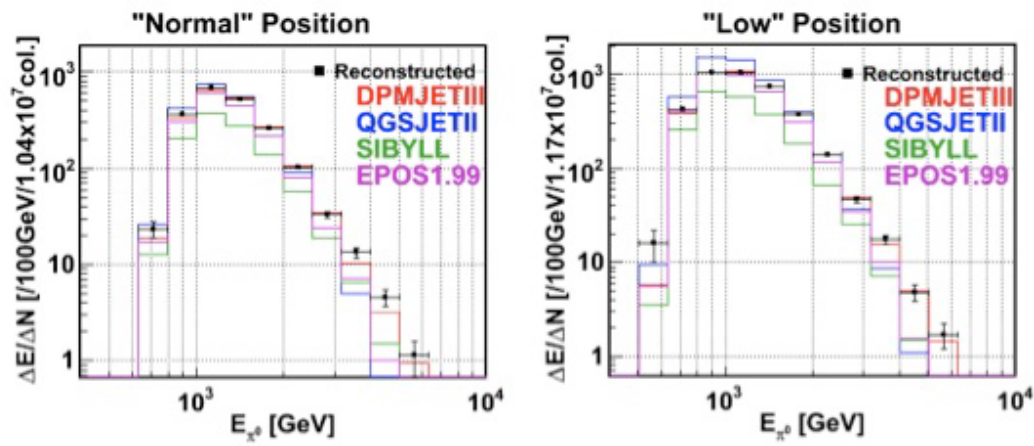

Figure 4: Reconstructed $\pi^{0}$ energy spectrum compared with expectations from different MC models for two detector positions. Events correspond to about 20 minutes $10^{29} \mathrm{~cm}^{-2} \mathrm{~s}^{-1}$ luminosity. The reconstructed spectrum well reproduce the production spectrum. The main systematic error is due to the uncertainty on the absolute energy scale of the calorimeter.
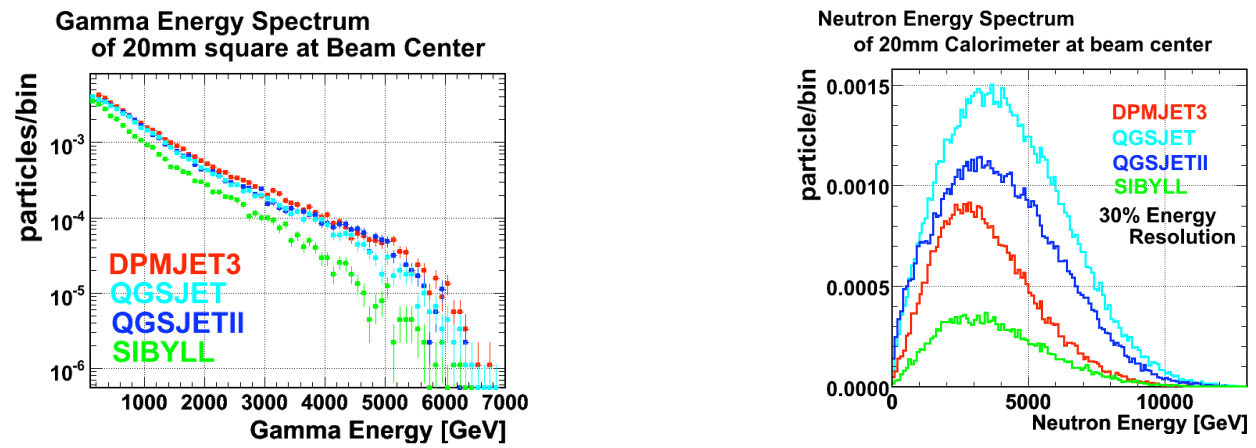

Figure 5: Expected energy spectrum for $\gamma \mathrm{s}$ and neutrons according to different interaction models at 7+7 $\mathrm{TeV}$ center of mass energy. For neutrons a $30 \%$ energy resolution has been taken into account.

of cosmic ray Monte Carlo in an energy range wider than the one expected at the beginning of the project.
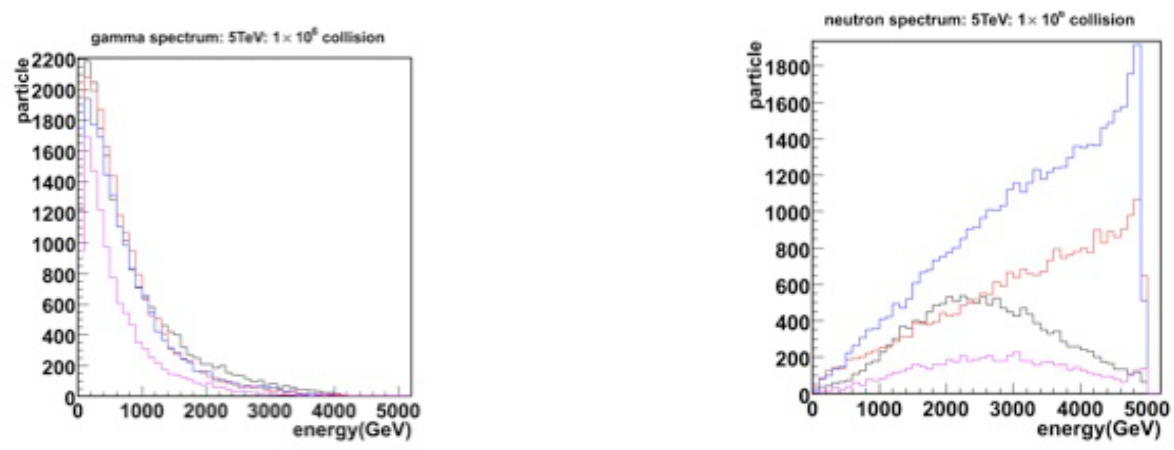

Figure 6: Expected energy spectrum for $\gamma \mathrm{s}$ (left) and neutrons (right) according to different interaction models for $5+5 \mathrm{TeV}$ proton-proton interaction. No detector resolution has been taken into account. The different colors show results for different MC codes: in blue DPMJET3, in red QGSJET2, in brown QGSJET1 and in purple SYBILL. 


\section{Conclusions}

The LHCf experiment at LHC is ready for data taking since January 2008 and will start its Physics program already with measurements in the beam commissioning phase of LHC. Thanks to the excellent detector performance for the reconstruction of photon, neutral meson and neutron spectra at different energies from $3.5+3.5 \mathrm{TeV}$ up to $7+7 \mathrm{TeV}$ proton runs, LHCf will be able to calibrate air shower Monte Carlo codes covering the most interesting energy range for HECR Physics, thus providing invaluable input to our understanding of high energy phenomena in the Universe.

\section{References}

[1] O. Adriani et al., The LHCf detector at the CERN Large Hadron Collider, JINST 3 (2008) S08006.

[2] M. Takeda et al., Extension of the cosmic ray energy spectrum beyond the predicted Greisen-Zatsepin-Kuz'min cutoff, Phys. Rev. Lett. 81 (1998) 1163 [astro-ph / 9807193 ].

[3] R. U. Abbasi et al., Measurement of the Flux of Ultrahigh Energy Cosmic Rays from Monocular Observations by the High Resolution Fly's Eye Experiment, Phys. Rev. Lett. 92 (2004) 1511.

[4] T. Yamamoto et al., The UHECR spectrum measured at the Pierre Auger Observatory and its astrophysical implications, Proc. of 30th International Cosmic Ray Conference, Merida, Mexico (2007) [arXiv: 0707.2638 [astro-ph]].

[5] The Pierre Auger Collaboration, Correlation of the Highest Energy Cosmic Rays with nearby Extragalactic Objects, Science 318 (2007) 938.

[6] M. Unger et al., Study of the Cosmic Ray Composition above $0.4 \mathrm{EeV}$ using the Longitudinal Profiles of Showers observed at the Pierre Auger Observatory, Proc. of 30th International Cosmic Ray Conference, Merida, Mexico (2007) [arXiv : 0706.1495v1 [astro-ph]]

[7] E. Paré et al., Inclusive production of $\pi^{0} \mathrm{~s}$ in the fragmentation region at the SppS collider, Phys. Lett. B 242 (1990) 531.

[8] O. Adriani et al., Technical design report of the LHCf experiment, CERN-LHCC-2006-004.

[9] H. Menjo et al., Simulation Study for the performance of the LHCf experiment, Proc. of 31st International Cosmic Ray Conference, Łódź, Poland (2009).

[10] S. Roesler et al., The Event generator DPMJET-III at cosmic ray energies, Proc. of 27th International Cosmic Ray Conference, Hamburg, Germany (2001).

[11] S. Ostapchenko, Status of QGSJET, AIP Conf. Proc. 928 (2007) 118 [arXiv: 0706.3784 [astro-ph]].

[12] R. S. Fletcher et al., SIBYLL: An Event generator for simulation of high-energy cosmic ray cascades, Phys. Rev. D 50 (1994) 5710.

[13] K. Werner and T. Pierog, Extended Air Shower Simulations Based on EPOS, AIP Conf. Proc. 928 (2007) 111 [arXiv: 0707.3330 [ astro-ph]]. 